\title{
MUTANTS OF Streptomyces cattleya DEFECTIVE IN THE SYNTHESIS OF A FACTOR REQUIRED FOR THIENAMYCIN PRODUCTION
}

\author{
Tim Buchan*, Claudia Roach, Carolyn Ruby ${ }^{\dagger}$, Dean Taylor, \\ Carol Preisig and Christopher Reeves \\ Panlabs, Inc. \\ Bothell, WA 98011 , U.S.A. \\ ${ }^{\dagger}$ Merck Research Laboratories, \\ Rahway, NJ 07065, U.S.A.
}

(Received for publication April 8, 1994)

\begin{abstract}
Thienamycin non-producing mutants of Streptomydes cattleya were identified that displayed a cross-feeding relationship. A diffusible product from one of these mutants (RK-11) resulted in restoration of thienamycin production when fed to cultures of another mutant (RK-4). In vivo radiolabeling experiments were conducted to test whether the RK-11 mutant produced a late biosynthetic intermediate which contained a carbapenem ring and a cysteaminyl and/or a hydroxyethyl side chain. Both $\left[{ }^{35} \mathrm{~S}\right]$ cystine and $\left[\right.$ methyl- $\left.{ }^{3} \mathrm{H}\right]$ methionine were used to label the RK-11 product which was then fed to RK-4 cultures. None of the thienamycin subsequently produced by RK-4 converter cells was labeled, implying the lack of either side chain of the thienamycin molecule in the RK-11 product. Further stability studies suggested that the RK-11 product does not contain a carbapenem ring. Additional feeding experiments with RK-4 cells also ruled out the possibility that the RK-11 product is a co-factor necessary for thienamycin production. It is concluded that the RK-11 product may regulate expression of the thienamycin gene cluster.
\end{abstract}

Thienamycin is one of the most potent, broad spectrum antibiotics presently known. It was the first discovered among a series of naturally produced $\beta$-lactams which contain the carbapenem ring system ${ }^{17}$. Elucidation of carbapenem biosynthesis lags behind that of the better known classes of $\beta$-lactams such as the penicillins and cephalosporins because of the technical difficulties associated with low titers and chemical instabilities. However, feeding studies with radiolabeled precursors have established the origin of all carbon atoms in the thienamycin molecule ${ }^{2)}$. Both the C-8 and C-9 atoms in the hydroxyethyl side chain are derived from the $S$-methyl group of methionine. The same studies also show that cystine, but not cysteamine or pantetheine, is incorporated into the cysteaminyl side chain (Fig. 1). Based on these results, a biosynthetic pathway for thienamycin has been proposed ${ }^{3)}$.

Streptomyces cattleya is one of only two species of Streptomyces known to produce thienamycin ${ }^{4}$. In order to obtain more information about the thienamycin biosynthetic pathway, non-producing mutants of the wild type S. cattleya (ATCC 35852) were isolated by screening for lack. of growth inhibition zones on a lawn of indicator bacteria. These mutants were characterized in the hopes of finding some which were blocked in the thienamycin biosynthetic pathway. One set of mutants showed an ordered pattern of cross-feeding and was selected for further study. Here we show that one mutant of

Fig. 1. Structure of the thienamycin molecule.

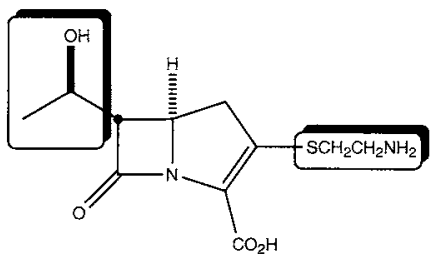

The hydroxyethyl side chain and the cysteaminyl side chain in relationship to the bicyclic carbapenem ring are indicated by the left and right-hand boxes, respectively. 
this set is deficient in the synthesis of a diffusible factor which, although structurally unrelated to thienamycin, is required for thienamycin biosynthesis. It does not appear to be an enzyme cofactor, but may be an activator of transcription of one or more thienamycin biosynthesis genes.

\section{Materials and Methods}

\section{Characterization of Mutants and Cross-Feeding Assays}

Streptomyces cattleya MA4297 was mutagenized with EMS (ethyl methanesulfonate). Colonies that did not produce thienamycin were identified by the failure to produce inhibition zones when plated on agar containing Staphylococcus aureus (ATCC 6538p) ${ }^{5}$. Pairs of mutants were streaked in a T-pattern on SBL agar plates and overlaid with $S$. aureus to establish cross-feeding relationships which restored thienamycin production. Cross-feeding occurred when a mutant blocked at an early step in the pathway converted an intermediate produced by a mutant blocked late in the pathway, resulting in thienamycin production (Fig. 2). Control experiments were performed to verify that the resulting inhibition zones were due to thienamycin production and not the result of other antibiotics produced by $S$. cattleya. As the result of such cross-feeding experiments, mutants RK-4 and RK-11 were selected for further study.

In a typical cross-feeding experiment in liquid medium, RK-4 and RK-11 mutants were grown for 24 hours in each of two seed media, and then transferred to synthetic production medium for 48 to 72 hours $^{3)}$. Following three washes in $50 \mathrm{mM} \mathrm{NaPO} 4 \mathrm{pH} 6.5$, each strain was resuspended to a $4 \times$ concentration in this buffer. This method was sufficient to prepare cells of the putative converter strain (RK-4) for cross-feeding. The putative producer strain (RK-11) was incubated an additional 24 hours at $28^{\circ} \mathrm{C}$ and $220 \mathrm{rpm}$. Following this incubation, the RK-11 cells were centrifuged at $4,000 \times g$ for 10 minutes and the supernatant passed through a series of sterile filters $(8.0 \sim 0.2 \mu \mathrm{M})$.

To evaluate cross-feeding, $125 \mu \mathrm{l}$ of a fresh RK-4 resting cell suspension and $500 \mu \mathrm{l}$ of the filtered $\mathrm{RK}-11$ supernatant were placed in sterile $16 \times 125 \mathrm{~mm}$ test tubes and incubated overnight at $28^{\circ} \mathrm{C}$ and $220 \mathrm{rpm}$. Cross-fed cultures were spun at $1,500 \times g$ and $50 \mu \mathrm{l}$ of the supernatant was placed on a paper antibiotic assay disc. After drying, the discs were transferred to a bioassay plate $(24.5 \mathrm{~cm} \times 24.5 \mathrm{~cm} \times 2 \mathrm{~cm})$ prepared from $200 \mathrm{ml}$ of LB agar containing $25 \mathrm{mg}$ of 2,3,5-triphenyltetrazolium chloride (TTC) and seeded with $800 \mu \mathrm{l}$ of an overnight $S$. aureus culture ${ }^{6}$. After overnight incubation at $37^{\circ} \mathrm{C}$, successful cross-feeding produced clear, colorless zones of growth inhibition around the discs.

\section{Isolation of RK-11 Product}

The RK-11 mutant was used to prepare a resting culture as described above ${ }^{3)}$. After incubation as a resting culture for 24 hours, the cells were spun at $16,000 \times g$ for 10 minutes, the pellet was discarded, and the supernatant was passed through a series of sterile filters $(8.0 \sim 0.2 \mu \mathrm{M})$. This filtrate was fractionated by reverse phase chromatography on Diaion HP-20 resin equilibrated with Milli-Q purified water. Fractions containing the RK-11 product were determined by the liquid cross-feeding assay described above. Active fractions from the column were pooled and concentrated by rotary evaporation. This concentrated pool was subjected to another round of HP-20 chromatography, after which fractions containing the RK-11 product were again identified by cross-feeding and $S$. aureus bioassay. The RK-11 product purified in this manner could then be used in radio-labeling studies or lyophilized for later HPLC analysis.

Stability of Thienamycin and RK-11 Product and High Temperature and Alkaline pH

To test the stability of thienamycin at alkaline $\mathrm{pH}$, the $\mathrm{pH}$ of a $500 \mathrm{~mm}$ thienamycin stock solution was adjusted to 12.0 with $\mathrm{NaOH}$ and the solution immediately placed at $32^{\circ} \mathrm{C}$. Upon addition of the $\mathrm{NaOH}$ and thereafter at 5 minute intervals, $25 \mu \mathrm{l}$ aliquots were removed and placed in tubes containing $25 \mu \mathrm{l}$ of $50 \mathrm{mM} \mathrm{NaPO}_{4}$ buffer, $\mathrm{pH} 6.5$ to neutralize the reactions. Contents of these tubes were later spotted onto dises and bioassayed on a $S$. aureus/TTC plate. After overnight incubation at $37^{\circ} \mathrm{C}$, the size of the inhibition zones were measured. The amount of thienamycin present could then be estimated by comparison to a standard curve.

To test the stability of the RK-11 product, a $900 \mu \mathrm{l}$ aliquot was taken from a pool partially purified by HP-20 chromatography. The $\mathrm{pH}$ of this aliquot was adjusted to 12.0 with $\mathrm{NaOH}$ and the aliquot placed 
at $32^{\circ} \mathrm{C}$. At 5 minute intervals, $125 \mu \mathrm{l}$ samples were taken from the incubation tube and placed in tubes containing $125 \mu \mathrm{l}$ of $50 \mathrm{~mm} \mathrm{NaPO}$ buffer, $\mathrm{pH} 6.5$, to neutralize the reactions. The tubes were stored on ice until all time points were taken. The contents of each tube were then cross-fed with a fresh $4 \times$ concentrated RK-4 resting cell suspension. After overnight incubation at $28^{\circ} \mathrm{C}$ and centrifugation, $50 \mu \mathrm{l}$ aliquots of each cross-feed were assayed for antibiotic activity using the $S$. aureus/TTC bioassay. The amount of thienamycin was again estimated by measuring inhibition zone sizes.

Stability to Hydroxylamine

Following 2 rounds to HP-20 chromatography and lyophilization, RK-11 product was resuspended in $240 \mu 1100 \mathrm{~mm}$ MOPS pH 7.0 plus $10 \mu 10.2 \mathrm{M}$ hydroxylamine $-\mathrm{HCl}$ and allowed to incubate at room temperature for 30 minutes to determine its stability to hydroxylamine. After incubation, the sample was fractionated by HPLC $\left(3.9 \times 300 \mathrm{~mm} \mathrm{C}{ }_{18}\right.$ Resolve cartridge, mobile phase $10 \mathrm{mM} \mathrm{H}_{3} \mathrm{PO}_{4}-\mathrm{KH}_{2} \mathrm{PO}_{4} \mathrm{pH}$ 3.5 at $1 \mathrm{ml} /$ minute). As a reference, another portion of the same RK-11 batch was resuspended in $250 \mu \mathrm{l}$ $0.2 \mathrm{M} \mathrm{H}_{3} \mathrm{PO}_{4}-\mathrm{KH}_{2} \mathrm{PO}_{4}$ buffer $\mathrm{pH} 3.05$. After a 30 minute incubation at room temperature, this sample was also resolved by HPLC using the same column conditions. During HPLC of both samples, $1 \mathrm{ml}$ fractions were collected into tubes containing $15 \mu \mathrm{l}$ of $500 \mathrm{mM} \mathrm{K} \mathrm{K}_{2} \mathrm{PO}_{4}$ neutralizing buffer. From each fraction, a $500 \mu \mathrm{l}$ aliquot was used for cross-feeding to $125 \mu \mathrm{l}$ of $4 \times$ concentrated RK -4 cells. After overnight incubation, $50 \mu \mathrm{l}$ aliquots of these cross-fed cultures were assayed for antibiotic activity.

Precursor Incorporation Studies

In experiments to evaluate the incorporation of the known thienamycin precursor, cystine, into the RK-11 product, a stock solution was prepared by dissolving $24 \mathrm{mg} \mathrm{L}$-cystine in $80 \mathrm{ml}$ of $\mathrm{H}_{2} \mathrm{O}$ and the pH was adjusted to 6.5 with $0.1 \mathrm{~N} \mathrm{NaOH}$. To this solution was added $46.2 \mu \mathrm{Ci} \mathrm{L}-\left[{ }^{35} \mathrm{~S}\right]$ cystine giving a final concentration of $1.25 \mathrm{~mm}$ cystine. Two $500 \mathrm{ml}$ baffled flasks each containing $40 \mathrm{ml}$ of $4 \times$ concentrated RK- 11 resting cells and $20 \mathrm{ml}$ of the cystine stock solution were incubated at $28^{\circ} \mathrm{C}$ and $220 \mathrm{rpm}$ for 6 hours, after which another $20 \mathrm{ml}$ of the stock solution was added and incubation was continued for 18 hours. The RK-11 product was partially purified by two cycles of HP-20 chromatography as described above. This partially purified product was cross-fed to a $4 \times$ concentration of RK-4 cells. After overnight incubation, the cells were spun at $16,000 \times g$ for 10 minutes and the supernatant decanted. Following careful adjustment to $\mathrm{pH} 7.0$ with $0.5 \mathrm{M} \mathrm{NH}{ }_{4} \mathrm{OH}$, the supernatant was fractionated by Dowex anion exchange chromatography $\left(\mathrm{HCO}_{3} \text {-form, AG-X2; } 50 \sim 100 \text { mesh }\right)^{2}$. The column was washed with one volume of cold $\mathrm{H}_{2} \mathrm{O}$ and the thienamycin was eluted with $\mathrm{CO}_{2}$-saturated $\mathrm{H}_{2} \mathrm{O}$. Both absorbance at $297 \mathrm{~nm}$ and bioassays on $S$. aureus/ TTC plates were used to determine the fractions containing thienamycin, which were then pooled and lyophilized. The lyophilized material was suspended in $10 \mathrm{mM} \mathrm{KHPO}_{4}$ buffer and the thienamycin further purified by HPLC $\left(8.9 \times 300 \mathrm{~mm} \mathrm{C}_{18}\right.$ Resolve column, mobile phase $10 \mathrm{~mm}$ $\mathrm{KHPO}_{4}$ at a flow rate of $1 \mathrm{ml} /$ minute). One milliliter fractions were collected, and aliquots of each fraction were counted on a Beckman liquid scintillation counter. Separate aliquots of each fraction were also tested for the presence of thienamycin using the $S$. aureus/TTC assay.

In another facet of the experiment, $\left[{ }^{35} \mathrm{~S}\right]$ cystine was added to semipurified $\mathrm{RK}-11$ product and this mixture was incubated overnight with RK-4 converter cells. The resulting thienamycin was partially purified by Dowex and HP-20 chromatographies, followed by fractionation by HPLC on Resolve $\mathrm{C}_{18}$. The HPLC fractions were both counted to determine $\left[{ }^{35} \mathrm{~S}\right]$ content and bioassayed for antibiotic activity as described above.

In a parallel series of experiments to evaluate the incorporation of another known thienamycin precursor, methionine, into the RK-11 product, a stock solution was prepared by dissolving $162.5 \mathrm{mg}$ L-methionine in $80 \mathrm{ml}$ of $\mathrm{H}_{2} \mathrm{O}$. To this solution was added $105 \mu \mathrm{Ci}$ of $\left[\right.$ methyl ${ }^{3} \mathrm{H}$ ]methionine giving the stock solution a final methionine concentration of $13.4 \mathrm{~mm}$. RK-11 cultures were incubated overnight with $\left[\right.$ methyl- $\left.{ }^{3} \mathrm{H}\right]$ methionine and the RK-11 product isolated as described above. This product was cross-fed with RK-4 cells and the resulting thienamycin was purified as in the $\left[{ }^{35} \mathrm{~S}\right]$ cystine experiments. The converse experiment, in which RK-11 supernatant and $\left[\right.$ methyl- $\left.{ }^{3} \mathrm{H}\right]$ methionine were simultaneously incubated with RK-4 converter cells, was also conducted. 


\section{Results}

Several mutants of the Streptomyces cattleya strain MA4297 were generated by EMS treatment and found to have cross-feeding relationships which restored antibiotic production as outlined in Fig. 2. Of these mutants, RK-4 and RK-11 were selected for further investigation because they were prototrophic and retained the abilities to sporulate and produce cephamycin $\mathrm{C}$. The product of cross-feeding RK-11 and RK-4 was demonstrated to be thienamycin (Fig. 3). Upon analysis by HPLC, the bioactive product eluted under a peak corresponding to the same absorbance spectrum and retention time as authentic thienamycin. This bioactivity was also destroyed by incubation with porcine renal dehydropeptidase-1 (data not shown), as is thienamycin ${ }^{7)}$. In addition, the RK-11 product was sensitive to hydroxylamine, as

Fig. 2. Cross-feeding relationship and growth properties of characterized mutants.

\begin{tabular}{|c|c|c|c|c|}
\hline$? \longrightarrow \underset{\mathrm{C}}{\mathrm{MA6358}}$ & $\begin{array}{c}? \longrightarrow \\
\text { P } \\
\text { MA6359 } \\
\text { RK4 } \\
\text { RK14 }\end{array}$ & $\underset{\text { MA6357 }}{\longrightarrow}$ & $\begin{array}{l}\longrightarrow \\
\mathrm{A}\end{array}$ & $\begin{array}{l}\text { Lenamycin } \\
\text { duction }\end{array}$ \\
\hline Mutant & $\begin{array}{c}\text { Cross-feeding } \\
\text { class }\end{array}$ & Sporulation & $\begin{array}{l}\text { Cephamycin C } \\
\text { production }\end{array}$ & Prototrophy \\
\hline MA6358 & $\mathrm{C}$ & - & - & - \\
\hline MA6359 & $\mathrm{D}$ & - & + & - \\
\hline RK-4 \& -14 & D & + & + & + \\
\hline MA6357 & $\mathrm{B}$ & + & + & + \\
\hline MA6361 & A & - & + & + \\
\hline RK-3, $-11 \&-12$ & A & + & + & + \\
\hline
\end{tabular}

See text for explanation.

Fig. 3. Thienamycin production in a RK-11 cross-feeding experiment.

A; A partially purified RK-11 product was incubated overnight with RK-4 cells. The resulting supernatant was analyzed by HPLC as described in Materials and Methods. Thienamycin was detected as a $297 \mathrm{~nm}$ absorbing peak at 17.27 minutes. The corresponding photodiode array spectrum from $200 \sim 400 \mathrm{~nm}$ is shown. B; A comparable HPLC analysis of a thienamycin standard is also shown.

A
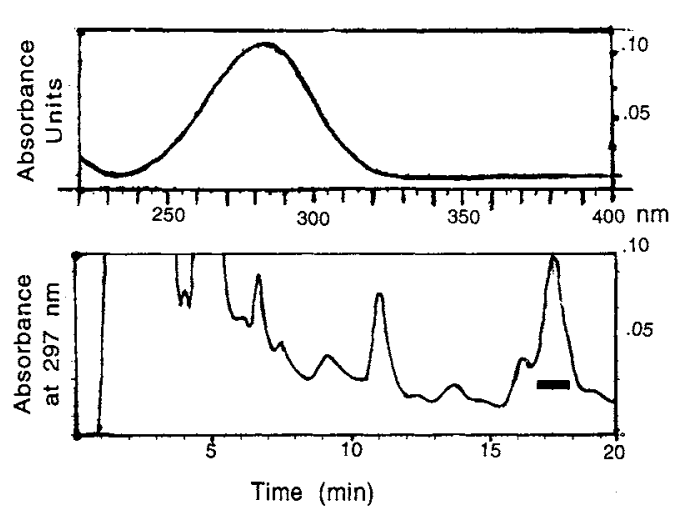

B

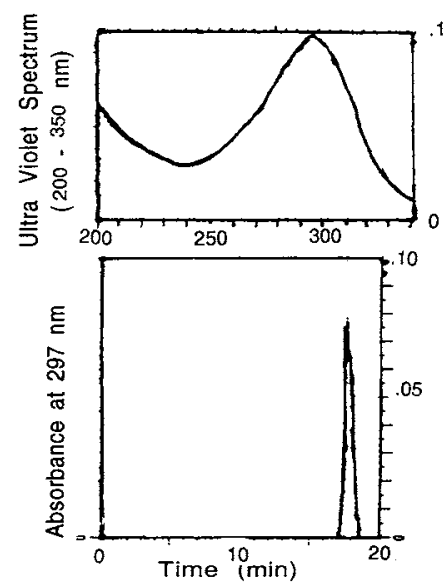


is thienamycin, suggesting that the two might be related (Fig. 4).

To determine whether these thienamycin non-producing mutants were blocked in thienamycin biosynthesis, we asked whether the RK-11 product could be labeled in vivo by feeding with $\left[{ }^{35} \mathrm{~S}\right]$ cystine or $\left[\right.$ methyl $\left.-{ }^{3} \mathrm{H}\right]$ methionine, both of which efficiently label thienamycin when added to wild type cultures ${ }^{3)}$. $\left[{ }^{35} \mathrm{~S}\right]$ Cystine is incorporated into the cysteaminyl side chain, while $\left[\right.$ methyl $\left.{ }^{3} \mathrm{H}\right]$ methionine is the source of both carbons of the hydroxylethyl side chain (Fig. 1). In both labeling experiments, the RK-11 product was partially purified from the radiolabeled cultures in order to remove unincorporated amino acid, and then the RK-11 product was added to a RK-4 culture. After overnight incubation, the resulting thienamycin was partially purified and analyzed for the extent of radioisotope incorporation.

In first experiment, RK-11 cells were labeled with $\left[{ }^{35} \mathrm{~S}\right]$ cystine and the RK-11 product was partially purified by two cycles of HP-20 chromatography, then incubated with RK-4 cells. The resulting thienamycin was purified from the cross-fed cultures by Dowex-bicarbonate chromatography, followed by HPLC on a Resolve $\mathrm{C}_{18}$ cartridge. No radioactivity copurified with the thienamycin in spite of the significant amount of thienamycin that was produced in this cross-feeding. In another version of this experiment, RK-11 supernatant and $\left[{ }^{35} \mathrm{~S}\right]$ cystine were added simultaneously to the RK-4 converter cells and incubated over night. In

Fig. 4. The effect of hydroxylamine on the RK-11 product.

A; A supernatant containing partially-purified RK-11 product after incubation with hydroxylamine was fractionated by Resolve C-18 HPLC. None of the fractions showed bioactivity after cross-feeding with RK-4 cells. B; Results of HPLC fractionation of a supernatant resulting from a cross-feed of RK-4 cells and partially-purified RK-11 product without hydroxylamine treatment are shown. Fractions showing bioactivity after cross-feeding are indicated by black bars.

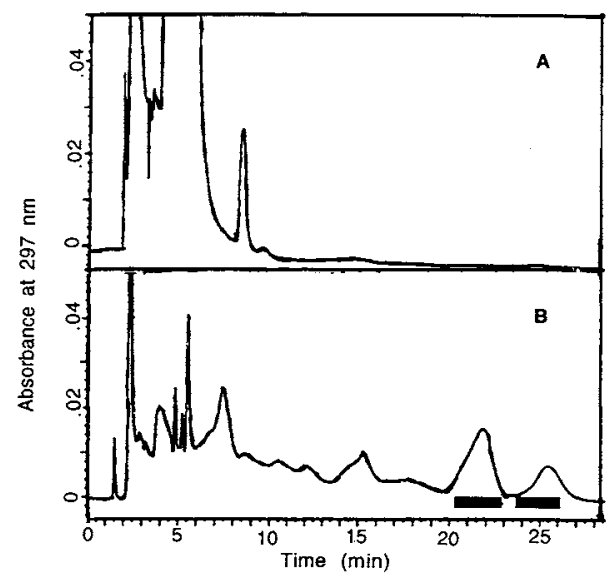

Fig. 5. Analysis of $\left[{ }^{35} \mathrm{~S}\right]$ cystine-labeling of $\mathrm{RK}-11$ product.
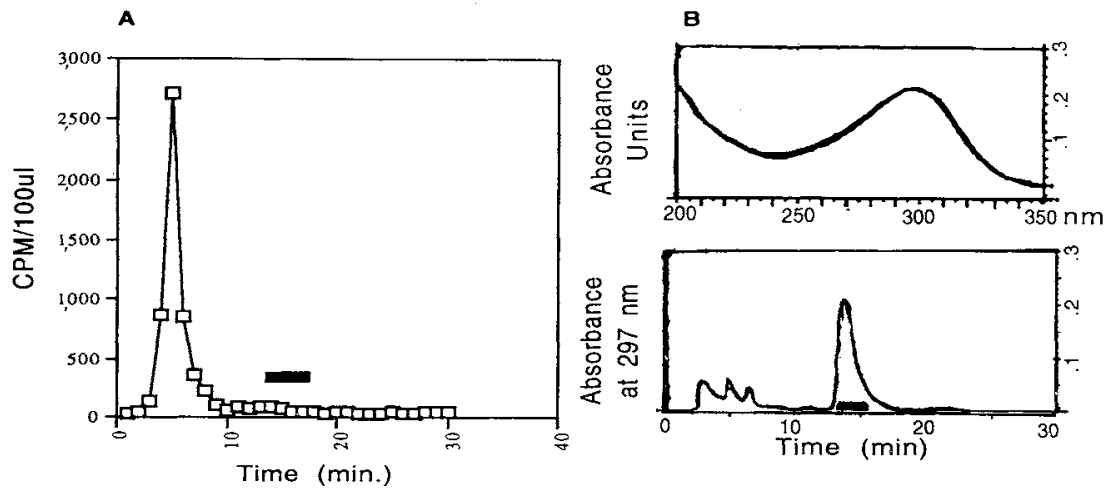

A culture supernatant was obtained following overnight incubation of RK-4 cells with isolated RK-11 product from $\left[{ }^{35}\right.$ S $]$ cystine labeled cultures. Thienamycin was purified from the culture supernatant by Dowex bicarbonate chromatography and Resolve C-18 HPLC as described in Materials and Methods. The radioactivity (A) and absorbance (B) profiles from the HPLC are shown. The black bars identify fractions which were bioactive. 
Fig. 6. Analysis of RK-4 culture supernatant following overnight incubation with RK-11 product and $\left[{ }^{35} \mathrm{~S}\right]$ cystine.
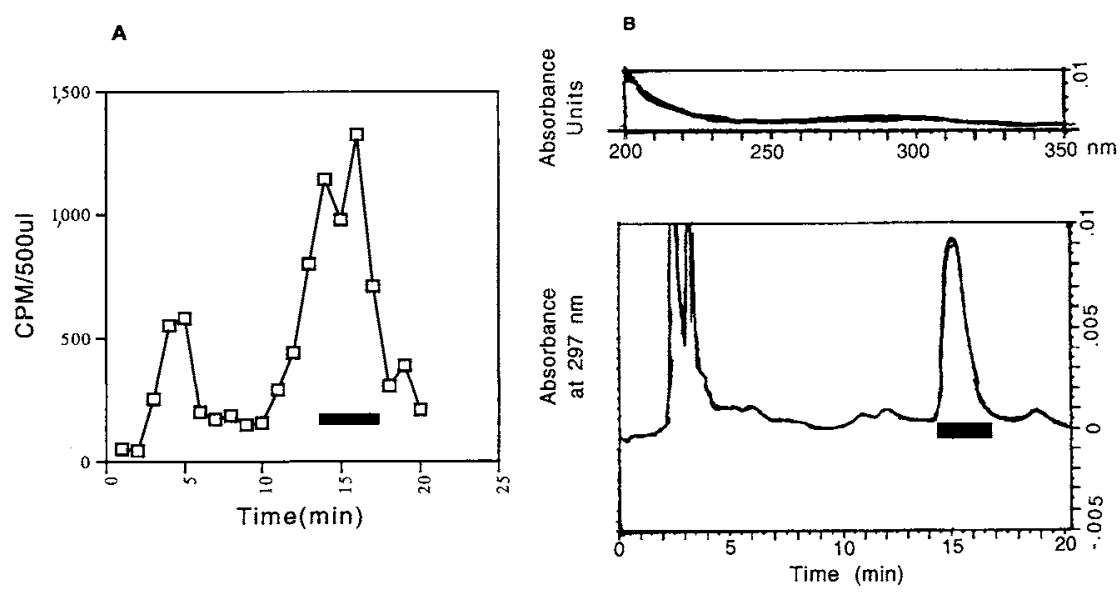

Thienamycin was purified from the cross-feed supernatant as described in Fig. 5 and Materials and Methods. The radioactivity (A) and absorbance (B) profiles from HPLC are shown with bioactive fractions indicated by black bars.

Fig. 7. Analysis of $\left[\right.$ methyl $\left.^{3} \mathrm{H}\right]$ methionine-labeling of RK-11 product.
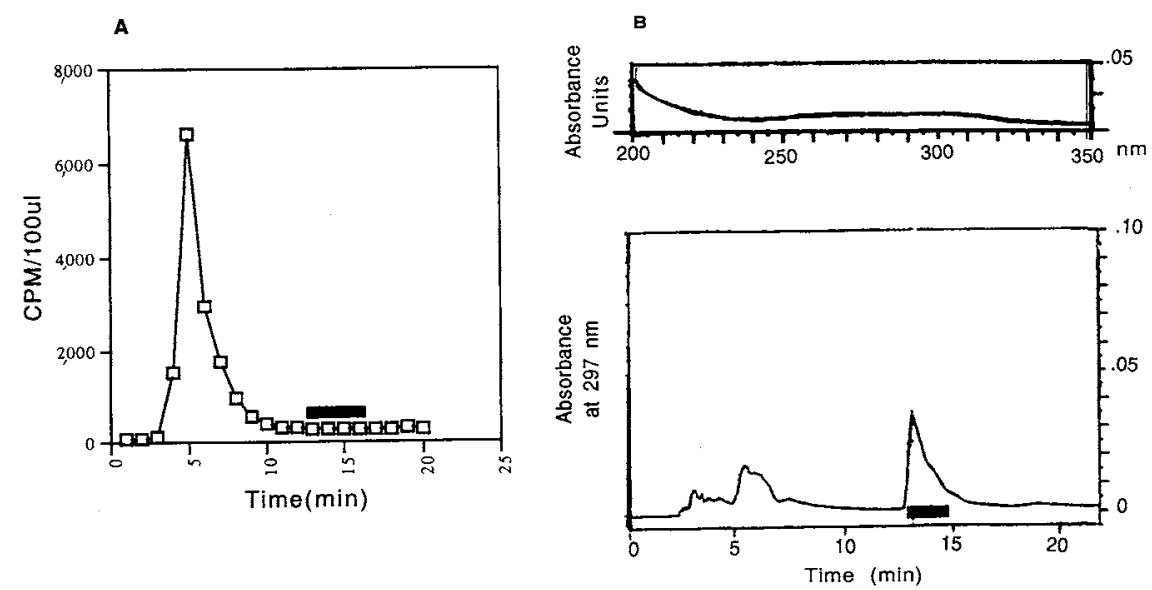

A culture supernatant was obtained following overnight incubation of RK-4 cells with isolated RK-11 product from $\left[\right.$ methy $\left./{ }^{3} \mathrm{H}\right]$ methionine labeled cultures. Thienamycin was purified from the cross-feed supernatants described in Fig. 5 and Materials and Methods. The radioactivity (A) and absorbance (B) profiles from HPLC are shown with the bioactive fractions indicated by black bars.

this case, substantial radioactivity copurified with the thienamycin (Fig. 6), indicating that the conditions used can result in the production of labeled thienamycin. These data indicate that the RK-11 product does not contain a C-2 cysteaminyl side chain (Fig. 1).

In the parallel feeding experiments with $\left[\right.$ methyl $\left.-{ }^{3} \mathrm{H}\right]$ methionine, $\mathrm{RK}-11$ product was isolated from a labeled culture. When this product was incubated with RK-4 cells and the resulting thienamycin was purified, no radiolabel was associated with it (Fig. 7). On the other hand, adding $\left[\right.$ methyl- $\left.{ }^{3} \mathrm{H}\right]$ methionine together with RK-11 culture supernatant to RK-4 cells resulted in radiolabeled thienamycin (Fig. 8). Together, these data indicate that the RK-11 product contains neither the hydroxyethyl side chain nor 
Fig. 8. Analysis of RK-4 culture supernatant following overnight incubation with RK-11 product and $\left[\right.$ methyl- $\left.{ }^{3} \mathrm{H}\right]$ methionine.
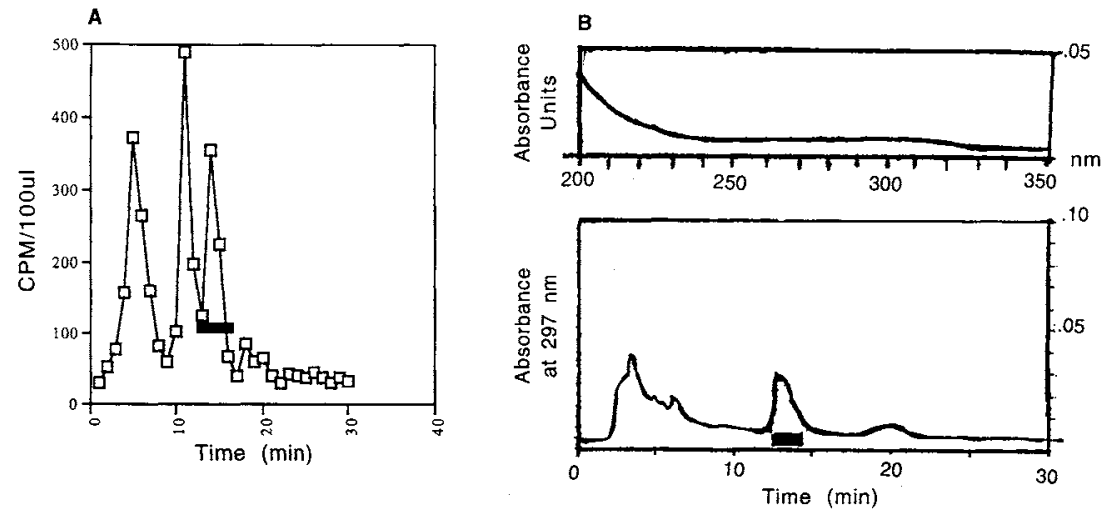

Thienamycin was purified from the cross-feed supernatant as described in Fig. 5 and Materials and Methods. The radioactivity (A) and absorbance profiles from HPLC (B) are shown with bioactive fractions indicated by black bars.

Table 1. The effect of extremes of temperature and $\mathrm{pH}$ on thienamycin and $\mathrm{RK}-11$ product.

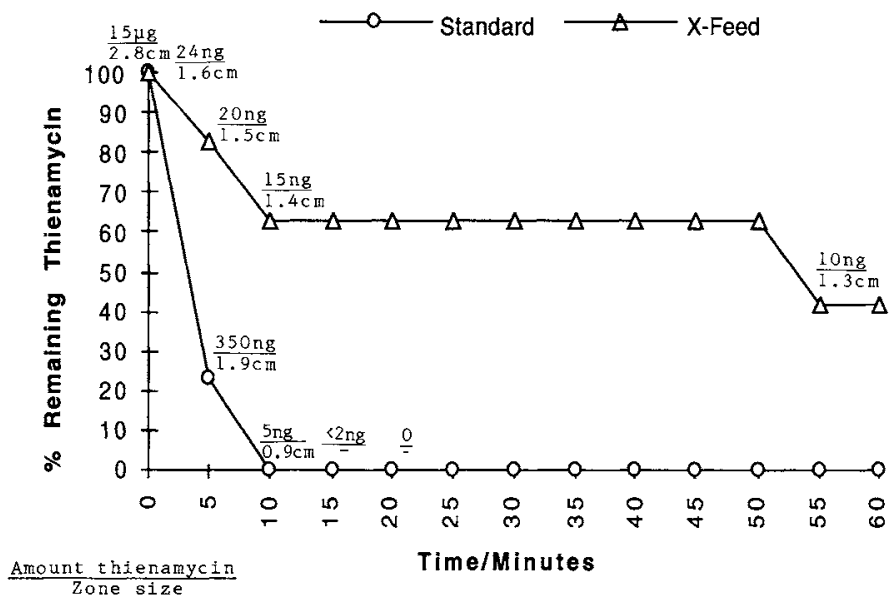

(O) Thienamycin standards were incubated at $32^{\circ} \mathrm{C}$ and $\mathrm{pH} 12.0$ for the amount of time indicated, then bioassayed on $S$. aureus/TTC plates, and the resulting inhibition zones measured. $(\triangle)$ Semi-purified RK-11 product was incubated at $32^{\circ} \mathrm{C}$ and $\mathrm{pH} 12.0$ for the time intervals indicated. After neutralization, the solution was cross-fed to fresh RK-4 cells. Thienamycin production was determined by inhibition zone sizes on $S$. aureus/TTC plates.

a carbon moiety constructed by successive methylations, as would be expected of most thienamycin intermediates.

In further characterization of the RK-11 product, we have also shown that the RK-11 product is more stable to $\mathrm{pH}$ and temperature extremes than is thienamycin. When thienamycin is exposed to high temperatures $\left(32^{\circ} \mathrm{C}\right)$ and $\mathrm{pH} 12.0$, it rapidly degrades, losing it's bioactivity (Table 1). In contrast, when partially purified RK-11 product is exposed to the same extremes of temperature and $\mathrm{pH}$ and then incubated with RK-4 cells, the ability to induce thienamycin production is more persistent (Table 1). Since the simple carbapenem structure, as produced by Serratia and Erwinia species, is even less stable than thienamycin 
to these conditions ${ }^{8)}$, it is unlikely that the RK-11 product contains a bicyclic carbapenem ring structure minus the two side chains (Fig. 1). In addition, the RK-11 product cannot be recovered from Dowexbicarbonate resin, a procedure that works well to purify thienamycin (data not shown). Since our data indicates that the RK-11 product is lacking a cysteaminyl side chain, a hydroxyethyl side chain, and the carbapenem ring structure, we conclude that the RK-11 product is neither structurally related to, nor a precursor of, thienamycin. Our data does indicate however, that the RK-11 product was able to stimulate de novo synthesis of thienamycin by RK-4 cells.

\section{Discussion}

We have characterized a set of mutants and shown that they are deficient in the synthesis of a factor required for thienamycin biosynthesis. Evidence presented here indicates that this factor is not a precursor of thienamycin, nor does it appear to have any structural relationship to thienamycin. Attempts to purify the RK-11 product to the extent necessary for structure determination were hampered by a non quantitative assay and the poor chemical stability of the product. Thus, we have been unable to obtain structural data for this factor and can only speculate as to its nature. One possiblity is that it is a precursor to a vitamin or cofactor required for an enzyme in the thienamycin pathway. Although RK-4 is not an auxotroph, suggesting it is not deficient in cofactor synthesis, the availability and requirement for cofactors may change during antibiotic production. We tested vitamin B12 and several other metabolites including pantetheine, biotin, and lipoic acid without finding any that induced RK-4 to produce thienamycin. Although unlikely, it is possible that the RK-11 product is a cofactor specific for thienamycin biosynthesis.

Another possibility is that the RK-11 product could be a molecule that regulates expression of thienamycin biosynthetic genes or a precursor of such a molecule. There are several examples of small molecules that regulate secondary metabolism in Streptomyces and other microorganisms ${ }^{9}$. Recently, a homoserine lactone has been shown to regulate carbapenem production in Erwinia carotovora ${ }^{10}$. We found that there is a threshold above which further additions of purified RK-11 product to RK-4 converter cells does not increase thienamycin production (data not shown), which is consistent with a regulatory function for the RK-11 product. We tested two commercialiy available autoregulators, A-factor and nonalactone, neigher of which induced RK-4 cells to produce thienamycin (data not shown). However, the structure of this potential regulator could be quite different.

It is curious that, of the many non-producing mutants which cross-fed with other mutants to yield thienamycin, none have been shown to be blocked in the biosynthetic pathway. This appears to be in opposition to work with $S$. fulvoviridis where cross-feeding and complementation have been successful in establishing a set of mutants which are blocked in the biosynthetic pathways of the OA-6129 group of carbapenems ${ }^{11}$. This suggests either that thienamycin is synthesized on a multifunctional complex and that the intermediates in the pathway are extremely labile, or that none of these intermediates are capable of diffusing between cells. More work on the biosynthetic pathway should resolve the question.

Acknowledgment

The authors would like to thank Dr. JoAnne WiLLIamson of Merck Research Laboratories for helpful discussions. We also thank PAM FRANKLIN for help in preparing this manuscript.

\section{References}

1) Kahan, J. S.; F. M. Kahan, R. Geogelman, S. A. Currie, M. Jackson, E. O. Stapley, T. W. Miller, D. Henduin, S. Mochales, S. Herandez, H. R. WoodrufF \& J. Birnbaum: Thienamycin, a new $\beta$-lactam antibiotic. I. Discovery, taxonomy, isolation, and physical properties. J. Antibiotics 32: $1 \sim 12,1979$

2) Treiber, L. R.; V. P. Gullo \& I. Putler: Procedure for isolation of thienamycin from fermentation broths. Biotechnology and Bioengineering 23: 1255 1265, 1981

3) Williamson, J. M.; E. Inamine, K. E. Wilson, A. W. Douglas, J. Liesch \& G. Albers-Schönberg: Biosynthesis of the $\beta$-lactam antibiotic thienamycin by Streptomyces cattleya. J. Biol. Chem. 260: 4637 4647, 1985 
4) Albers-Schönberg, G.; B. H. Areson, O. D. Hensens, K. Hoogsteen, E. A. Kaczha, R. E. Rhodes, J. S. Kahan, R. B. Morin \& B. G. Christensen: Structure and absolute configuration of thienamycin. J. Am. Chem. Soc. 100: $649 \mathrm{I} \sim 6499,1978$

5) FOOR, F.; B. TYLER \& N. MORIN: Effect of glycerol, phosphate, and pH on cellular differentiation and production of the beta-lactam antibiotic thienamycin by Streptomyces cattleya. Developments in Industrial Microbiol. 23: $305 \sim 313,1982$

6) Chen, C. W.; H. F. Lin, C. L. Kuo, H. L. Tsai \& J. F. Y. Tsai: Cloning and expression of a DNA sequence conferring cephamycin C production. Biotechnology 6: 1222 1224, 1988

7) Kroff, H.; J. G. Sundelof, R. Hadju \& F. M. KaHN: Metabolism of thienamycin and related antibiotics by the renal dipeptidase dehydropeptidase-1. Antimicrob. Agents Chemother. 22: 62 70, 1982

8) Bycroft, B. W.; C. MASLen, S. J. Box, A. G. Brown \& J. W. Tyler: The isolation and characterization of $(3 R, 5 R)$ - and $(3 S, 5 R)$-carbapenam-3-carboxylic acid from Serratia and Erwinia species and their putative biosynthetic role. J. Chem. Soc., Chem. Commun: $1623 \sim 1625,1987$

9) BepPU, T.: Secondary metabolites as chemical signals for cellular differentiation. Gene 115: 159 165, 1992

10) Bainton, N. J.; P. Stead, S. R. Chambra, B. W. Bycroft, G. P. C. Salmond, G. Steward \& P. Williams: $N$-(3-Oxohexanoyl)-L-homoserine lactone regulates carbapenem antibiotic production in Erwinia carotovora. Biochem. J. 288: $997 \sim 1005,1992$

11) Nakata, K.; S. Horinouchi \& T. Beppu: Cloning and characterization of the carbapenem biosynthetic genes from Streptomyces fulvoviridis. FEMS Microbiol. Lett. 57: 51 55, 1989 